\title{
Identification of MAGEA12 as a prognostic outlier gene in gastric cancers
}

\author{
J. WU ${ }^{1}$, J. WANG ${ }^{1}$, W. SHEN ${ }^{2, *}$ \\ ${ }^{1}$ The Criminal Science and Technology Department, Zhejiang Police College, Hangzhou, China; ${ }^{2}$ The First Affiliated Hospital of Zhejiang Chinese \\ Medical University, Hangzhou, China.
}

*Correspondence: weishenzj@sina.com

Received July 19, 2016 / Accepted October 22, 2016

\begin{abstract}
Melanoma antigen (MAGE) family genes are frequently over-expressed in a subset population of multiple cancers, and serve as idea therapeutic targets; however, their distribution pattern in gastric cancers has not yet been evaluated. In this study, we first performed a cancer outlier profile analysis (COPA) on a series of public gene expression datasets of gastric cancer, and identified MAGEA12 showing a significant outlier expression model reproducibly. We further in silico validated that MAGEA12 outlier over-expression were associated with poor clinical outcome using six microarray datasets from GEO database. We then experimentally detected the MAGEA12 expression in an independent cohort of gastric cancer samples by immunohistochemistry, and showed that over-expression of MAGEA12 in a subset of cancers was associated with later stage and reduced survival; furthermore, MAGEA12 was an independent prognostic factor in an outlier manner. Our results indicate that MAGEA12 is a novel prognostic outlier gene in gastric cancers and patterns of MAGE expression may inform individualized targeted immunotherapies.
\end{abstract}

Key words: gastric cancer, MAGE, MAGEA12, prognosis, outlier

Gastric cancer is one of the most common malignancies worldwide [1]. However, its treatment has evolved relatively little, especially for cases at the late stages. Currently, the efficient therapeutic options which could improve the outcome of patients are very limited. Therefore, identification of novel therapeutic molecular targets has important clinical and biological implications.

The melanoma antigen genes (MAGE) were initially isolated from melanomas and turned out to be a family of cancer-testis antigens (CTA) including over fifty closely related MAGE proteins [2]. MAGE derived peptides can be recognized cytolytic $\mathrm{T}$ lymphocytes and cause tumor rejection, making MAGE antigens as idea targets. A series of clinical trials with MAGE antigens are in progress $[3,4]$. Previous studies have identified several MAGE members up-regulated in gastric cancers and associated with more aggressive phenotypes and poor clinical outcomes [5-7]. Most of MAGE proteins are not expressed in normal tissues except testis, however, only a few MAGE antigens were identified over-expressed by comparing the gene expression profiles of gastric cancer and normal gastric tissues. Therefore, we hypothesized that some MAGE members may express in an outlier manner, that is these genes only up-regulated in a subset, but not all of gastric cancers.

In this study, using a cancer outlier profile analysis (COPA) approach, we investigated the outlier expression pattern of a list of MAGE members from the GEO gastric cancer gene expression datasets. We identified that MAGEA12 demonstrated an outlier expression model reproducibly, and the outlier over-expression model was associated with poor clinical outcome. Then we further validated the clinicpathological and prognostic significance of MAGEA12 outlier over-expression in an independent cohort of gastric cancers by immunohistochemistry.

\section{Materials and methods}

Cancer outlier profile analysis (COPA). To nominate MAGE family members with marked over-expression in a subset of cases from the transcriptomic data, the Cancer outlier profile analysis (COPA) algorithm was performed using five expression gastric cancer mRNA expression microarray data- 
sets (GSE22377, GSE15456, GSE14208, GSE15459, GSE15081) in GEO database as described before [8]. GEO database is the largest fully public repository for microarray and other type high-throughput data, primarily gene expression data. Briefly, the expression value from microarray datasets were median centered, the median value of each gene was set to 0. A COPA score was calculated by dividing each gene expression value by its median absolute deviation (MAD). Then genes were ranked based on their COPA scores and outlier genes were defined if they ranked in the top scores at the $75^{\text {th }}, 90^{\text {th }}$ or $95^{\text {th }}$ cutoffs. Genes showing outlier expression across multiple studies were further ranked according to the scores in a significant fraction $\left(\mathrm{p}<1 \times 10^{-5}\right)$ of datasets using Meta-COPA analysis. Both COPA and meta-COPA were implemented using the web-based procedure on the Oncomine database [9].

Survival analysis of MAGEA12 outlier over-expression in six independent gene expression microarray datasets of gastric cancer. We next analyzed the correlation between MAGEA12 outlier over-expression with free progression survival (FPS) and overall survival (OS) in six independent gastric cancer gene expression cohorts deposited in GEO database (GSE14120, GSE15459, GSE22377, GSE29272, GSE51105, and GSE62254). Based on the $75^{\text {th }}$ expression value, cases were divided into MAGEA12 outlier over-expression (high) and low expression subgroups. Then the Kaplan-Meier survival plots were used to compare the difference in FPS and OS between the two distinct subgroups in combined cohorts as described previously [10].

Clinical gastric cancer specimens for immunohistochemical evaluation. A cohort of 162 cases of archival formalin-fixed, paraffin-embedded gastric cancer tissues was used to experimentally validate the clinical and prognostic significance of MAGEA12. The cancerous and paired adjacent normal specimens were collected from patients who underwent radical resection from May 2007 to April 2010. All the patients received standard treatment according to the Chinese guidelines for gastric cancer management. The follow-up time were determined between surgical resection and cancer-related death, ranged from 3 to 96 months. No patients received neoadjuvant radio-chemotherapy. The use of clinical samples and the study procedures were reviewed and approved by our individual institutional ethical committees.

Immunohistochemistry (IHC). IHC was performed on gastric cancer and normal gastric sections for the determination of MAGEA12 expression. Briefly, sections ( $4 \mu \mathrm{m}$ thick) were de-paraffinizated and rehydrated. Endogenous peroxidases activity was quenched in $3 \% \mathrm{H} 2 \mathrm{O} 2$ for 10 min at room temperature. Antigen retrieval was treated in $10 \mathrm{mM}$ citrate buffer at $\mathrm{pH} 6.0$ for $10 \mathrm{~min}$. Then sections were incubated with a rabbit anti-human MAGEA12 antibody (Abcam, 1:150 dilution) overnight at $4^{\circ} \mathrm{C}$, followed by the incubation of a HRP-conjugated secondary antibody substrate (Dako, Copenhagen, Denmark). Diaminobenzidine was used as the chromogen. Non-specific rabbit immunoglobulin G was used as the negative control. A semi-quantitative IHC score method (H score) was used for data analysis [11]. The method assigned a score to each cases based on the percentage of cells stained at different intensities. 75 percentile score were set as the discriminatory threshold. Cases with an $\mathrm{H}$ score higher than this threshold were classified into MAGEA12 outlier overexpression (high), and the others as low expression.

Statistics. Correlation between MAGEA12 expression status and clinical characteristics was assessed by chi square test. Kaplan-Meier plot was used to compare the difference in survival between different subgroups, and evaluated by logrank test. In multivariate analysis, each variable was tested in a Cox model for proportional hazards. $\mathrm{P}<0.05$ was considered as statistically significant. The statistical analyses were performed using MedCalc software for Windows, Version 15.8.

\section{Results}

Using the COPA algorithm, we nominated and ranked the MAGE family member genes across five gastric cancer gene expression datasets with marked expression in a subset, but not all of the cases. Our meta-COPA analysis identified a list of outlier genes, and MAGEA12 is the most significant outlier gene in the list (Table 1). As seen in Table 2, MAGEA12

Table 1. Meta-COPA analysis on the expression of MAGE family genes across microarray datasets

\begin{tabular}{lcccccccc}
\hline \multirow{2}{*}{ Gene } & \multicolumn{2}{c}{ Outlier 75th\% } & \multicolumn{2}{c}{ Outlier 90th\% } & \multicolumn{2}{c}{ Outlier 95th\% } & \multicolumn{2}{c}{ otal } \\
\cline { 2 - 8 } & Median Rank & COPA Score & Median Rank & COPA Score & Median Rank & COPA Score & Median Rank & COPA Score \\
\hline MAGEA12 & 4 & 5.037 & 53 & 5.316 & 161 & 7.007 & 61 & 1.518 \\
MAGEA10 & 2149 & 1.269 & 151 & 3.831 & 99 & 9.677 & 151 & 3.831 \\
MAGEA2 & 268 & 1.656 & 80 & 4.295 & 190 & 5.181 & 175 & 353.319 \\
MAGEA3 & 14 & 3.416 & 204 & 3.816 & 212 & 5.082 & 204 & 3.186 \\
MAGEA4 & 5942.5 & 1.325 & 239.5 & 138.802 & 218.5 & 166.991 & 308 & 4.36 \\
MAGEA5 & 1285.5 & 41.81 & 205.5 & 4.084 & 616.5 & 4.814 & 330 & 4.454 \\
MAGEA6 & 25 & 2.653 & 415 & 3.699 & 1360.5 & 4.102 & 337 & 4.164 \\
MAGEA9 & 3977 & 2.154 & 123 & 3.348 & 211 & 7.42 & 561 \\
MAGEB2 & 1110 & 1.621 & 492 & 3.612 & 454 & 158.046 & 570 & 3.533 \\
TRO & 1354 & 4.847 & 1614 & 2.828 & 3524 & 3.396 & 9.211 \\
\hline
\end{tabular}



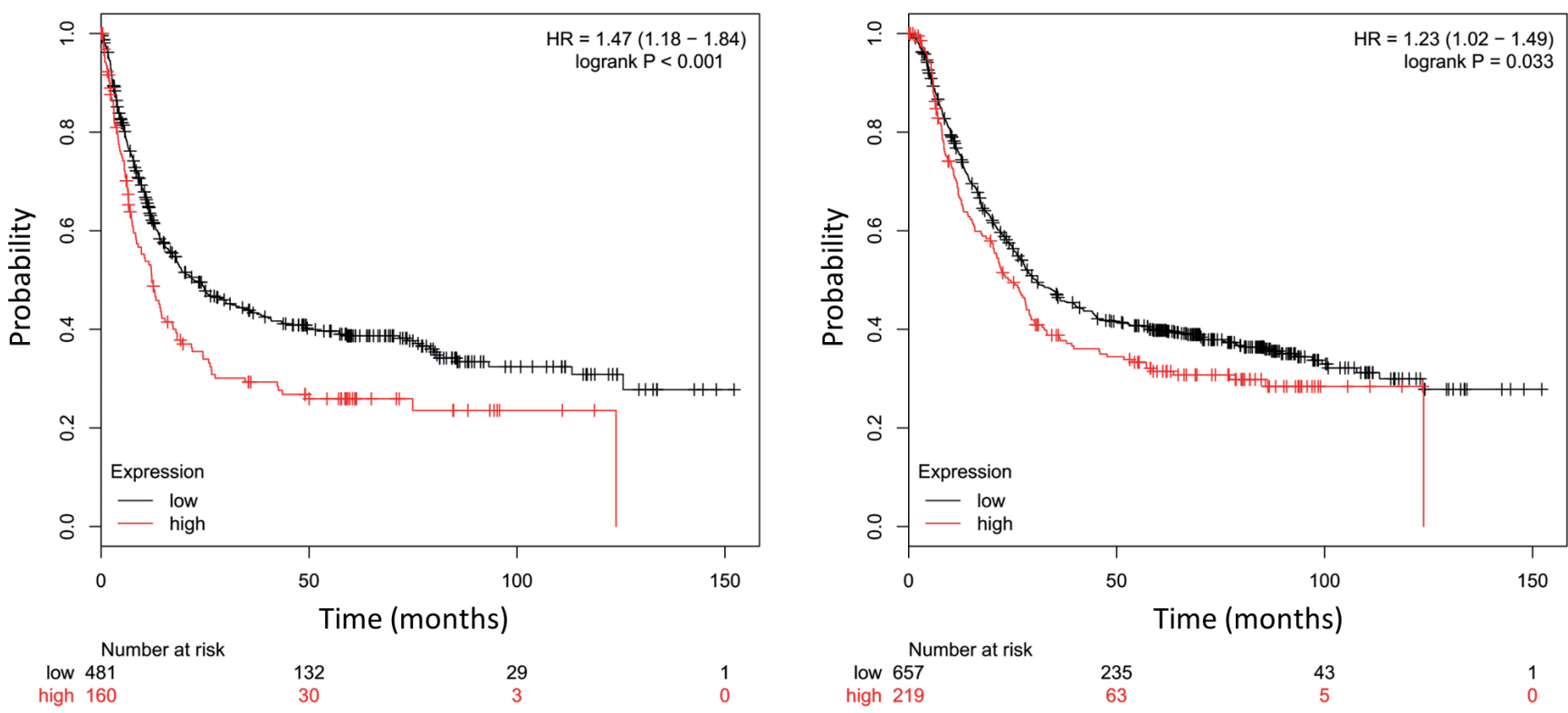

Figure 1. Kaplan-Meier plots comparing free-progression (A) and overall survival (B) in cases with and without MAGEA12 outlier over-expression in the combined six gene expression microarray datasets.

demonstrated a typical outlier model in gastric cancer across different datasets and at all 75th, 90th and 95th percentile.

Next, we performed survival analysis on MAGEA12 outlier over-expression in six independent gastric cancer gene expression datasets. In COPA analysis, $75^{\text {th }}$ percentile demonstrated the highest RNAK and score compared with $90^{\text {th }}$ and $95^{\text {th }}$ percentile, therefore we selected $75^{\text {th }}$ percentile as a cut-off to define outlier MAGEA12 over-expression in the survival analysis. MAGEA12 outlier expression showed significant prognostic significance in GSE15459 dataset (FPS: $\mathrm{HR}=1.63,95 \% \mathrm{CI}=1.08-2.44, \mathrm{P}=0.018$; OS: $\mathrm{HR}=1.58,95 \% \mathrm{CI}=1.03-2.43, \mathrm{P}=0.034)$. Other datasets also indicate a trend between MAGEA12 outlier over-expression and shorter FPS or OS, but not reached a statistical significance. We further combined the six datasets into one, as seen Figure 1, MAGEA12 outlier over-expression retained to be a significant prognostic factor in the meta-analysis.

Furthermore, we experimentally evaluated the clinical and prognostic significance in a 162 gastric cancer cohort. Our IHC results showed that MAGEA12 was positively stained in the membrane and cytoplasm of cancer cells
(Figure 2), while no or weak staining was observed in adjacent normal gastric tissues. Our immunohistochemical results validated that MAGEA12 only stained in a minor proportion of gastric cancer tissues, demonstrating a typical outlier expression pattern. Using $75^{\text {th }}$ percentile $\mathrm{H}$ score as a cutoff, 41 cases were classified as MAGEA12 outlier over-expression (high). As seen in Table 3, we found that MAGEA12 outlier over-expression status was more frequently seen in tumors at late stages $(\mathrm{p}=0.048)$. Survival analysis using the IHC data demonstrated that MAGEA12 outlier expression was positively associated with reduced survival duration (Figure 3), and was independent of other clinically employed predictors in multivariate analysis (Table 4).

\section{Discussion}

MAGE antigens are highly specific to cancer cells and silent in normal tissues, thus representing a group promising targets. In this study, we found that part of the MAGE

Table 2. MAGEA12 outlier expression pattern in five microarray datasets

\begin{tabular}{|c|c|c|c|c|c|c|c|c|}
\hline \multirow{2}{*}{ Dataset } & \multirow{2}{*}{ Platform } & \multirow{2}{*}{ probe } & \multicolumn{2}{|c|}{ Outlier 75th\% } & \multicolumn{2}{|c|}{ Outlier 90th\% } & \multicolumn{2}{|c|}{ Outlier 95th\% } \\
\hline & & & COPA score & Rank & COPA score & Rank & COPA score & Rank \\
\hline GSE22377 & GPL570 & 210467_x_at & 1112.295 & 2 & 1215.01 & 11 & 1321.12 & 29 \\
\hline GSE15456 & GPL96 & 210467_x_at & 4.613 & 3 & 5.316 & 53 & 7.007 & 161 \\
\hline GSE14208 & GPL571 & 210467_x_at & 5.037 & 4 & 6.094 & 88 & 6.374 & 340 \\
\hline GSE15459 & GPL570 & 210467_x_at & 4.552 & 53 & 5.375 & 155 & 1.444 & 861 \\
\hline GSE15081 & GPL1291 & AGhsB110604 & 1.158 & 61 & 3.413 & 100 & 3.871 & 861 \\
\hline
\end{tabular}



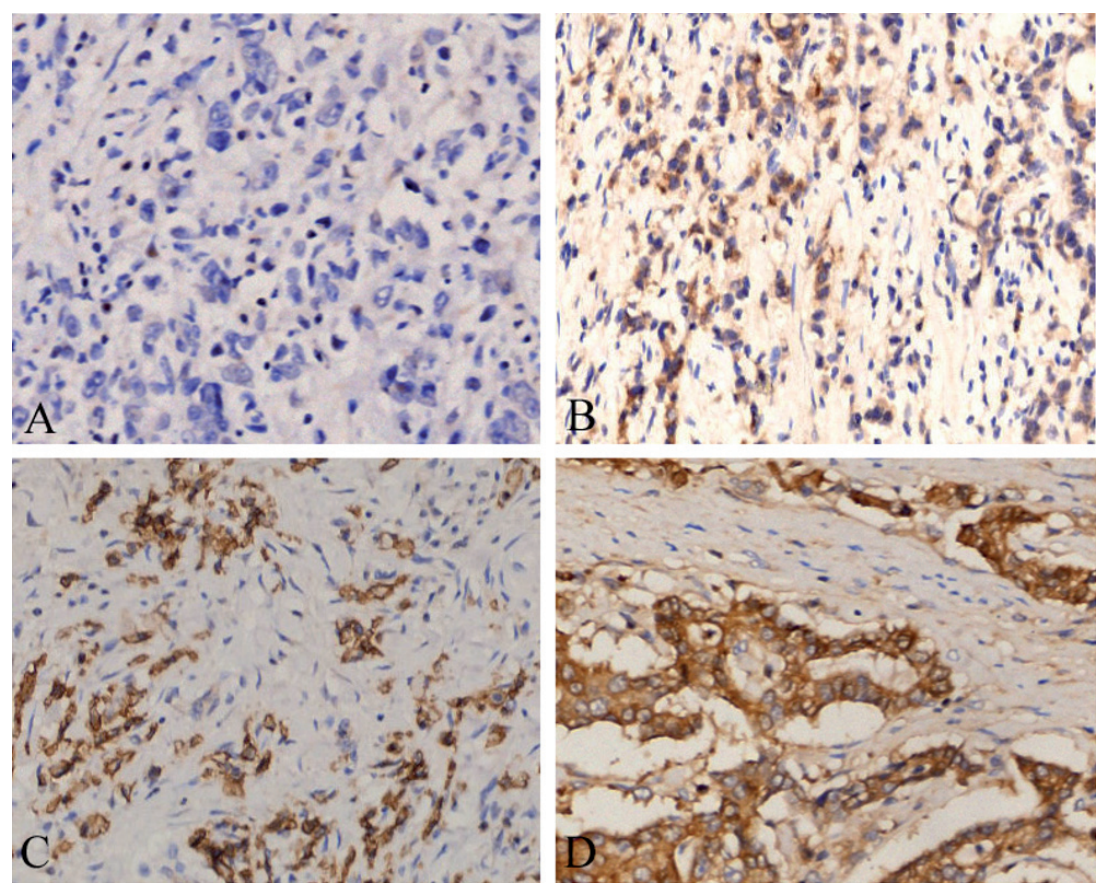

Figure 2. Representative images of negative MAGEA12 immunostaining in stage I (A) and positive staining in stage II (B), stage III (C), stage IV (D) in gastric cancer tissues.

Table 3. Clinical and pathological significance of MAGEA12 outlier expression in gastric cancer evaluated by IHC

\begin{tabular}{|c|c|c|c|}
\hline \multirow{2}{*}{ Characteristics } & \multicolumn{3}{|c|}{ MAGEA12 outlier expression } \\
\hline & High & Low & $\mathrm{P}$ \\
\hline \multicolumn{4}{|l|}{ Age } \\
\hline$<60$ years & 19 & 57 & 0.950 \\
\hline$\geq 60$ years & 22 & 69 & \\
\hline \multicolumn{4}{|l|}{ Gender } \\
\hline Male & 21 & 84 & 0.110 \\
\hline Female & 20 & 22 & \\
\hline \multicolumn{4}{|l|}{ Grade } \\
\hline 1 & 0 & 4 & \\
\hline 2 & 19 & 49 & 0.400 \\
\hline 3 & 22 & 73 & \\
\hline \multicolumn{4}{|l|}{ Tumor size } \\
\hline $\mathrm{T} 1-2$ & 3 & 23 & 0.150 \\
\hline $\mathrm{T} 3-4$ & 38 & 103 & \\
\hline \multicolumn{4}{|c|}{ Lymph node metastasis } \\
\hline Negative & 5 & 25 & 0.380 \\
\hline Positive & 36 & 101 & \\
\hline \multicolumn{4}{|c|}{ Distant metastasis } \\
\hline Negative & 38 & 121 & 0.650 \\
\hline Positive & 3 & 5 & \\
\hline \multicolumn{4}{|l|}{ Stage } \\
\hline 1 & 0 & 15 & \\
\hline 2 & 13 & 28 & 0.048 \\
\hline $3-4$ & 28 & 83 & \\
\hline
\end{tabular}

antigens, in particular MAGEA12, were not expressed universally in gastric cancer, but demonstrated an outlier expression pattern. In fact, previous studies have also demonstrated that MAGEA1, MAGEA2, MAGED2 expressed in only a minor proportion of gastric cancer tissues $(9.8 \%$ -

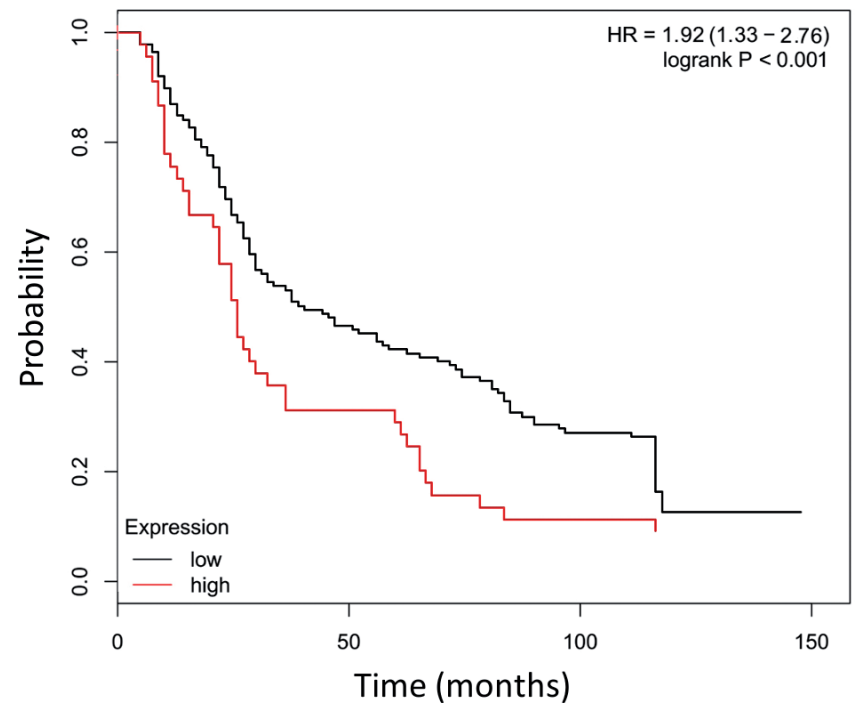

Figure 3. Kaplan-Meier plots comparing overall survival in a cohort of 162 gastric cancer cases with and without MAGEA12 outlier over-expression defined by immunohistochemistry. 
Table 4. Univariate and multivariate analysis for survival in terms of MAGEA12 outlier status evaluated by immunohistochemistry

\begin{tabular}{lcccc}
\hline \multirow{2}{*}{ Prognostic factors } & Univariate analysis & \multicolumn{3}{c}{ Multivariate analysis } \\
\cline { 2 - 5 } & HR $(95 \% \mathrm{CI})$ & $\mathrm{P}$ & HR $(95 \% \mathrm{CI})$ & $\mathrm{P}$ \\
\hline Grade & $1.28(0.97-1.68)$ & 0.083 & $1.22(0.90-1.65)$ & 0.200 \\
Tumor size & $2.74(1.71-4.38)$ & $<0.001$ & $1.94(1.18-3.20)$ & 0.009 \\
Lymph node metastasis & $2.29(1.48-3.53)$ & $<0.001$ & $1.66(1.06-2.61)$ & 0.029 \\
Distant metastasis & $3.20(1.54-6.64)$ & 0.002 & $2.96(1.40-6.24)$ & 0.005 \\
MAGEA12 outlier & $1.92(1.33-2.76)$ & $<0.001$ & $1.78(1.23-2.58)$ & 0.002 \\
\hline
\end{tabular}

45\%) [5-7]. In this study, using a meta-analysis approach on multiple gene expression datasets, we further validated that the outlier over-expression model of MAGE genes can be observed reproducibly.

For the first time, in this study, MAGEA12 overexpression was found to be linked to gastric cancer. Our data also suggest that only a small subset of gastric cancer patients may benefit from MAGEA12-targeted therapy, and the characteristics of MAGEA12 positive subgroup deserve further investigation. In this study, both our in silico analysis and IHC results support that MAGEA12 acts as an prognostic factor at both mRNA and protein levels, and gastric cancer patients with MAGEA12 outlier expression display a relatively poor clinical outcome and aggressive progressive behaviors. More meaningfully, the prognostic potential of MAGEA12 outlier expression is independent of other validated clinical predictors including tumor grade, size and metastasis status. Considering that MAGEA12 as a novel potential target for cancers [12], we propose that the subgroup of MAGEA12-positive expression gastric cancer cases demonstrating a relatively poor prognosis may benefit from MAGEA12 targeting treatment, and improve their clinical outcome.

Compare with other MAGE members, MAGEA12 has been seldom characterized in cancers. Yamada et al. [13] found that MAGEA12 showed high expression in cancer stem cells, a subgroup with a typical outlier distribution within tumors. Aberrant over-expression of MAGEA12 has been identified in oral squamous cell carcinoma and breast cancer $[14,15]$. Interestingly, MAGEA12 mRNA can be detected in the blood of $13 \%$ breast cancers, and may be used as a biomarker to monitor the progression and therapeutic effectiveness [15]. In this study, we further identified MAGEA12 expression as a novel predictor for the outcome of gastric cancers. Currently, several other prognostic markers in gastric cancer such as such as MUC1 have been validated [16], therefore, here we also proposed that combination of MAGEA12 with other factors may add prognostic and classification information for gastric cancers.

Immunotherapy against MAGEA12 is still at the early stage. Akiyama et al. [17] have identified a peptide from MAGEA12 (IFSKASEYL) as a novel CTL epitope. Based on our findings, we proposed that a subgroup of gastric cancer with MAGEA12 overexpression could be a candidate for MAGEA12 targeting immunotherapy in the future.

Acknowledgements: This study was support by the National Science Foundation of Zhejiang Province (LY14C010003) and the Hangzhou Scientific and Technological Program (20130432B07).

\section{References}

[1] SIEGEL R, MA J, ZOU Z, JEMAL A. Cancer statistics, 2014. CA Cancer J Clin 2014; 64: 9-29. https://doi.org/10.3322/ caac. 21208

[2] WEON JL, POTTS PR. The MAGE protein family and cancer. Curr Opin Cell Biol 2015; 37: 1-8. https://doi.org/10.1016/j. ceb.2015.08.002

[3] KRISHNADAS DK, SHUSTERMAN S, BAI F, DILLER L, SULLIVAN JE et al. A phase I trial combining decitabine/ dendritic cell vaccine targeting MAGE-A1, MAGE-A3 and NY-ESO-1 for children with relapsed or therapy-refractory neuroblastoma and sarcoma. Cancer Immunol Immunother 2015; 64: 1251-1260. https://doi.org/10.1007/s00262-015$\underline{1731-3}$

[4] LU YC, PARKER L, LU T, ZHENG Z, YAO X et al. A Phase I study of an HLA-DPB1* 0401-restricted T cell receptor targeting MAGE-A3 for patients with metastatic cancers. J Immunother Cancer 2015; 3(Suppl 2): 158. https://doi. org/10.1186/2051-1426-3-S2-P158

[5] KANDA M, NOMOTO S, OYA H, TAKAMI H, SHIMIZU $\mathrm{D}$ et al. The Expression of Melanoma-Associated Antigen D2 Both in Surgically Resected and Serum Samples Serves as Clinically Relevant Biomarker of Gastric Cancer Progression. Ann Surg Oncol 2016; 23(Suppl 2): S214-221. https:// doi.org/10.1245/s10434-015-4457-8

[6] JEON CH, KIM IH, CHAE HD. Prognostic value of genetic detection using CEA and MAGE in peritoneal washes with gastric carcinoma after curative resection: result of a 3-year follow-up. Medicine (Baltimore) 2014; 93: e83. https://doi. org/10.1097/MD.0000000000000083

[7] OGATA K, AIHARA R, MOCHIKI E, OGAWA A, YANAI M et al. Clinical significance of melanoma antigen-encoding gene-1 (MAGE-1) expression and its correlation with poor prognosis in differentiated advanced gastric cancer. Ann Surg Oncol 2011; 18: 1195-1203. https://doi.org/10.1245/s10434-010-1399-Z 
[8] MACDONALD JW, GHOSH D. COPA--cancer outlier profile analysis. Bioinformatics. 2006; 22: 2950-2951. https://doi. org/10.1093/bioinformatics/btl433

[9] RHODES DR, KALYANA-SUNDARAM S, MAHAVISNO V, VARAMBALLY R, YU J, BRIGGS BB et al. Oncomine 3.0: genes, pathways, and networks in a collection of 18,000 cancer gene expression profiles. Neoplasia 2007; 9: 166-180. https:// doi.org/10.1593/neo.07112

[10] SZASZ AM, LANCZKY A, NAGY A, FORSTER S, HARK $\mathrm{K}$ et al. Cross-validation of survival associated biomarkers in gastric cancer using transcriptomic data of 1,065 patients. Oncotarget 2016; [Epub ahead of print]

[11] SPECHT E, KAEMMERER D, SÄNGER J, WIRTZ RM, SCHULZ $S$ et al. Comparison of immunoreactive score, HER2/neu score and $\mathrm{H}$ score for the immunohistochemical evaluation of somatostatin receptors in bronchopulmonary neuroendocrine neoplasms. Histopathology 2015; 67: 368377. https://doi.org/10.1111/his. 12662

[12] HEIDECKER L, BRASSEUR F, PROBST-KEPPER M, GUEGUEN M, BOON T et al. Cytolytic T lymphocytes raised against a human bladder carcinoma recognize an antigen encoded by gene MAGE-A12. J Immunol 2000; 164: 6041-6045. https://doi.org/10.4049/jimmunol.164.11.6041
[13] YAMADA R, TAKAHASHI A, TORIGOE T, MORITA R, TAMURA Y et al. Preferential expression of cancer/testis genes in cancer stem-like cells: proposal of a novel sub-category, cancer/testis/stem gene. Tissue Antigens 2013; 81: 428-434. https://doi.org/10.1111/tan.12113

[14] MOLLAOGLU N, VAIRAKTARIS E, NKENKE E, NEUKAM FW, RIES J. Expression of MAGE-A12 in oral squamous cell carcinoma. Dis markers 2008; 24: 27-32. https://doi. org/10.1155/2008/359840

[15] ABD-ELSALAM EA, ISMAEIL NA. Melanoma-associated antigen genes: a new trend to predict the prognosis of breast cancer patients. Med Oncol 2014; 31: 285. https://doi. org/10.1007/s12032-014-0285-0

[16] WANG XT, KONG FB, MAI W, LI L, PANG LM. MUC1 Immunohistochemical Expression as a Prognostic Factor in Gastric Cancer: Meta-Analysis. Dis Markers 2016; 2016: 9421571. https://doi.org/10.1155/2016/9421571

[17] AKIYAMA Y, KOMIYAMA M, NAKAMURA Y, IIZUKA A, OSHITA C et al. Identification of novel MAGE-A6- and MAGE-A12-derived HLA-A24-restricted cytotoxic T lymphocyte epitopes using an in silico peptide-docking assay. Cancer Immunol Immunother 2012; 61: 2311-2319. https:// doi.org/10.1007/s00262-012-1298-1 